K. Hirata

Nagoya Math. J.

Vol. 33 (1968), 107-115

\title{
SOME TYPES OF SEPARABLE EXTENSIONS OF RINGS
}

\author{
KAZUHIKO HIRATA
}

\section{Introduction}

In [5] we defined the notion of separable extensions of a ring as a generalization of that of separable algebras. In this paper we try to introduce the notion of 'central' separable algebras to separable extensions. Such extensions are viewed in \$2. But we do not know that it is suitable or not to call them central separable extensions. As the Morita theorem for projective generators is fundamental to examine the central separable algebras we generalize it in $\S 1$. Let $R$ be a ring and let $A$ and $B$ be left $R$-modules. We assume that $R$-module $B$ is isomorphic to a direct summand of a finite direct sum of copies isomorphic to $A$. If $A=R$ then this means that $B$ is finitely generated projective, and if $B=R$ then $A$ is a generator. Let $S=\operatorname{End}_{R}(A)$ and $T=\operatorname{End}_{R}(B)$ be $R$-endomorphism rings of $A$ and $B$ respectively. Then $\operatorname{Hom}\left({ }_{R} A,{ }_{R} B\right)$ and $\operatorname{Hom}\left({ }_{R} B,{ }_{R} A\right)$ are finitely generated projective $S$-modules as well as generators as $T$-modules. Therefore if we further assume that $A$ is isomorphic to a direct summand of a finite direct sum of isomorphic copies of $B$ then we have an equivalence between the category of left (resp. right) $S$-modules and that of left (resp. right) $T$-modules.

In $\$ 2$ we consider a ring $A$ and its subring $\Gamma$. $A$ is said to be a separable extension of $\Gamma$ if there is an element $\sum a_{i} \otimes b_{i}$ in $\Lambda \otimes_{\Gamma} A$ such that $\sum a_{i} b_{i}=1$ and $\sum \lambda a_{i} \otimes b_{i}=\sum a_{i} \otimes b_{i} \lambda$ for all $\lambda \in \Lambda$. If we assume that the two-sided $\Lambda$-module $\Lambda \otimes_{\Gamma} \Lambda$ is isomorphic to a direct summand of a finite direct sum of copies of $\Lambda$ then we can prove that $\Lambda$ is a separable extension of $\Gamma$.

In $\S 3$ we consider a separable extension $\Gamma \otimes_{R} \Delta$ of $\Gamma$ where $\Delta$ is a central separable algebra over a commutative ring $R$. Then we can prove that the separable extension $\Gamma \otimes R \Delta$ of $\Gamma$ is of the type described in $\$ 2$.

Received March 7, 1968. 
Throughout this paper we assume that all rings have the identity and all subrings contain this element.

\section{\$1. A generalization of the Morita theorem}

In this section we use the notation that the homomorphisms from a module to the other or endomorphisms of a module operate on the opposite side of this module. Let $R$ be a ring and let $A$ and $B$ be two left $R$-modules. We set $S=\operatorname{End}_{R}(A)$ and $T=\operatorname{End}_{R}(B)$. Then $S$ and $T$ operate on the right of $A$ and $B$ respectively and we have bimodules ${ }_{R} A_{S},{ }_{R} B_{T}$, ${ }_{S} \operatorname{Hom}\left({ }_{R} A,{ }_{R} B\right)_{T}$ and ${ }_{T} \operatorname{Hom}\left({ }_{R} B,{ }_{R} A\right)_{S}$. Furthermore for $a \in A$ and $f \in \operatorname{Hom}\left({ }_{R} A,{ }_{R} B\right)$ the usual notation $f(a)$ is written by $a \cdot f$. Therefore if $f \in \operatorname{Hom}\left({ }_{R} A,{ }_{R} B\right)$ and $g \in \operatorname{Hom}\left({ }_{R} B,{ }_{R} A\right)$ then $f \cdot g \in S$ and $g \cdot f \in T$.

Now we assume that the $R$-module ${ }_{R} B$ is isomorphic to a direct summand of a finite direct sum of isomorphic copies of ${ }_{R} A$ and denote it by ${ }_{R} B\left\langle{ }_{R}(A \oplus \cdots \oplus A)\right.$. This means that if $A=R$ then $B$ is a finitely generated projective $R$-module, and, if $B=R$ then $A$ is a generator.

It is easily proved that ${ }_{R} B\left\langle\Theta_{R}(A \oplus \cdots \oplus A)\right.$ if and only if there exist a finite number of $g_{i} \in \operatorname{Hom}\left({ }_{R} B,{ }_{R} A\right)$ and $f_{i} \in \operatorname{Hom}\left({ }_{R} A,{ }_{R} B\right)$ such that $\sum g_{i} \cdot f_{i}=1_{B}$.

For modules $\operatorname{Hom}\left({ }_{R} B,{ }_{R} A\right)$ and $\operatorname{Hom}\left({ }_{R} A,{ }_{R} B\right)$ there is a homomorphism $\mu: \operatorname{Hom}\left({ }_{R} B,{ }_{R} A\right) \otimes \otimes_{S} \operatorname{Hom}\left({ }_{R} A,{ }_{R} B\right) \longrightarrow \operatorname{Hom}\left({ }_{R} B,{ }_{R} B\right)=T$ defined by $\mu(g \otimes f)$ $=g \cdot f$. By the above remark, the proof of next proposition is easy.

PROposition 1. 1. ${ }_{R} B\left\langle\Theta_{R}(A \oplus \cdots \oplus A)\right.$ if and only if $\mu$ is an epimorphism. If $\mu$ is an epimorphism, then $\mu$ is an isomorphism.

There is a homomorphism $\psi: \operatorname{Hom}\left({ }_{R} A,{ }_{R} B\right) \longrightarrow \operatorname{Hom}\left(\operatorname{Hom}\left({ }_{R} B,{ }_{R} A\right)_{S}, S_{S}\right)$ defined by $\phi(f)(g)=f \cdot g$ for $f \in \operatorname{Hom}\left({ }_{R} A,{ }_{R} B\right)$ and $g \in \operatorname{Hom}\left({ }_{R} B,{ }_{R} A\right)$. Similarly we have $\psi^{\prime}: \operatorname{Hom}\left({ }_{R} B,{ }_{R} A\right) \longrightarrow \operatorname{Hom}\left({ }_{S} \operatorname{Hom}\left({ }_{R} A,{ }_{R} B\right),{ }_{S} S\right)$ defined by $\psi^{\prime}(g)(f)=f \cdot g . \quad \psi$ is an $(S, T)$-homomorphism and $\psi^{\prime}$ is a $(T, S)$-homomorphism. Using $\psi$ we have a commutative diagram

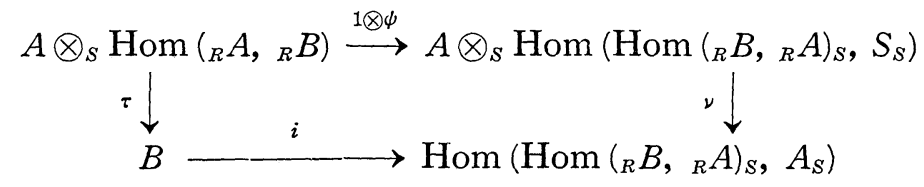

where $\tau, \quad i$ and $\nu$ are defined by $\tau(a \otimes f)=a \cdot f, \quad i(b)(g)=b \cdot g$ and $\nu(a \otimes h)(g)=a \cdot(h(g))$ respectively, for $a \in A, f \in \operatorname{Hom}\left({ }_{R} A,{ }_{R} B\right), \quad b \in B$, 
$g \in \operatorname{Hom}\left({ }_{R} B,{ }_{R} A\right)$ and $h \in \operatorname{Hom}\left(\operatorname{Hom}\left({ }_{R} B,{ }_{R} A\right)_{S}, S_{S}\right)$. These are all $(R, T)$ homomorphisms.

Theorem 1. 2. If ${ }_{R} B\left\langle\oplus_{R}(A \oplus \cdots \oplus A)\right.$, then we have:

(i) $\quad \psi$ and $\psi^{\prime}$ are isomorphisms.

(ii) All homomorphisms in the above diagram are isomorphisms.

(iii) $\operatorname{Hom}\left({ }_{R} A,{ }_{R} B\right)$ is finitely generated and projective as a left $S$-module as well as a generator as a right T-module. $\operatorname{Hom}\left({ }_{R} B,{ }_{R} A\right)$ is finitely generated and projective as a right $S$-module as well as a generator as $a$ left T-module.

(iv) $\operatorname{Hom}\left({ }_{S} \operatorname{Hom}\left({ }_{R} A,{ }_{R} B\right),{ }_{S} \operatorname{Hom}\left({ }_{R} A,{ }_{R} B\right)\right) \cong T$ and $\operatorname{Hom}\left(\operatorname{Hom}\left({ }_{R} B,{ }_{R} A\right)_{S}\right.$, $\left.\operatorname{Hom}\left({ }_{R} B,{ }_{R} A\right)_{S^{\prime}}\right) T$ as rings.

Proof. Let $g_{i} \in \operatorname{Hom}\left({ }_{R} B,{ }_{R} A\right)$ and $f_{i} \in \operatorname{Hom}\left({ }_{R} A,{ }_{R} B\right)$ be elements satisfying the condition $\sum g_{i} \cdot f_{i}=1_{B}$.

(i) Define $\psi^{-1}$ : Hom $\left(\operatorname{Hom}\left({ }_{R} B,{ }_{R} A\right)_{S}, S_{S}\right) \longrightarrow \operatorname{Hom}\left({ }_{R} A,{ }_{R} B\right)$ by $\psi^{-1}(h)$ $=\sum h\left(g_{i}\right) f_{i}$ for $h \in \operatorname{Hom}\left(\operatorname{Hom}\left({ }_{R} B,{ }_{R} A\right)_{S}, S_{S}\right)$. Then it is easily proved that $\psi^{-1} \cdot \psi$ and $\psi \cdot \psi^{-1}$ are identity maps respectively.

Remark. In Theorem A. 2 (f) [1], $\psi$ is an isomorphism, and so in Proposition A. 6, $\psi$ is an isomorphism without the assumption that $E$ is finitely generated projective.

(ii) Define $\alpha$ : Hom $\left(\operatorname{Hom}\left({ }_{R} B,{ }_{R} A\right)_{S}, A_{S}\right) \rightarrow A \otimes_{S} \operatorname{Hom}\left({ }_{R} A,{ }_{R} B\right)$ by $\alpha(k)=\sum k\left(g_{i}\right) \otimes f_{i}, k \in \operatorname{Hom}\left(\operatorname{Hom}\left({ }_{R} B,{ }_{R} A\right)_{S}, A_{S}\right)$. Then it is easy to prove that the composition maps from any term to itself is identity. For example, $(i \cdot \tau \cdot \alpha)(k)=(\nu \cdot(1 \otimes \psi) \cdot \alpha)(k)=\nu\left(\sum k\left(g_{i}\right) \otimes \psi\left(f_{i}\right)\right) \quad$ and $\quad \nu\left(\sum k\left(g_{i}\right) \otimes \psi\left(f_{i}\right)\right)(g)=$ $\sum k\left(g_{i}\right) \cdot \psi\left(f_{i}\right)(g)=\Sigma k\left(g_{i}\right) \cdot\left(f_{i} \cdot g\right)=\Sigma k\left(g_{i} \cdot f_{i} \cdot g\right)=k(g)$ for $g \in \operatorname{Hom}\left({ }_{R} B,{ }_{R} A\right)$.

(iii) Since ${ }_{R} B\left\langle\Theta_{R}(A \oplus \cdots \oplus A)\right.$ we have

$$
\begin{aligned}
{ }_{S} \operatorname{Hom}\left({ }_{R} A,{ }_{R} B\right) & \left\langle\oplus{ }_{S} \operatorname{Hom}\left({ }_{R} A,{ }_{R}(A \oplus \cdots \oplus A)\right)\right. \\
& \cong{ }_{S}\left(\operatorname{Hom}\left({ }_{R} A,{ }_{R} A\right) \oplus \cdots \oplus \operatorname{Hom}\left({ }_{R} A,{ }_{R} A\right)\right)
\end{aligned}
$$

and

$$
\begin{aligned}
\operatorname{Hom}\left({ }_{R} B,{ }_{R} B\right)_{T} & \left\langle\oplus \operatorname{Hom}\left({ }_{R}(A \oplus \cdots \oplus A),{ }_{R} B\right)_{T}\right. \\
& \cong\left(\operatorname{Hom}\left({ }_{R} A,{ }_{R} B\right) \oplus \cdots \oplus \operatorname{Hom}\left({ }_{R} A,{ }_{R} B\right)\right)_{T} .
\end{aligned}
$$

Therefore $\operatorname{Hom}\left({ }_{R} A,{ }_{R} B\right)$ is finitely generated projective as a left $S$-module and a generator as a right $T$-module. Similarly for $\operatorname{Hom}\left({ }_{R} B,{ }_{R} A\right)$. 
(iv) We have following isomorphisms

$$
\begin{aligned}
& \operatorname{Hom}\left({ }_{S} \operatorname{Hom}\left({ }_{R} A,{ }_{R} B\right),{ }_{S} \operatorname{Hom}\left({ }_{R} A,{ }_{R} B\right)\right) \\
\cong & \operatorname{Hom}\left({ }_{S} \operatorname{Hom}\left({ }_{R} A,{ }_{R} B\right),{ }_{S} S\right) \otimes_{S} \operatorname{Hom}\left({ }_{R} A,{ }_{R} B\right) \\
\cong & \operatorname{Hom}\left({ }_{R} B,{ }_{R} A\right) \otimes{ }_{S} \operatorname{Hom}\left({ }_{R} A,{ }_{R} B\right) \cong \operatorname{Hom}\left({ }_{R} B,{ }_{R} B\right)=T .
\end{aligned}
$$

The first isomorphism is a consequence of finiteness and projectivity of $S$ module $\operatorname{Hom}\left({ }_{R} A,{ }_{R} B\right)$, the second is given by $\psi^{\prime} \otimes 1$ and the last is Proposition 1. 1. This is given explicitly by $t \longrightarrow(f \longrightarrow f \cdot t)$ for $t \in T$ and $f \in \operatorname{Hom}\left({ }_{R} A,{ }_{R} B\right)$. Similarly we have

$$
\begin{aligned}
& \operatorname{Hom}\left(\operatorname{Hom}\left({ }_{R} B,{ }_{R} A\right)_{S}, \operatorname{Hom}\left({ }_{R} B,{ }_{R} A\right)_{S}\right) \\
\cong & \operatorname{Hom}\left({ }_{R} B,{ }_{R} A\right) \otimes \otimes_{S} \operatorname{Hom}\left(\operatorname{Hom}\left({ }_{R} B,{ }_{R} A\right)_{S}, S_{S}\right) \\
\cong & \operatorname{Hom}\left({ }_{R} B,{ }_{R} A\right) \otimes_{S} \operatorname{Hom}\left({ }_{R} A,{ }_{R} B\right) \cong \operatorname{Hom}\left({ }_{R} B,{ }_{R} B\right) .
\end{aligned}
$$

This is given by $t \longrightarrow(g \longrightarrow t \cdot g)$ for $t \in T$ and $g \in \operatorname{Hom}\left({ }_{R} B,{ }_{R} A\right)$. This completes the proof of Theorem 1. 2.

Remark. If the map $i$ is an isomorphism and $\operatorname{Hom}\left({ }_{R} B,{ }_{R} A\right)$ is right $S$-finitely generated and projective then we have ${ }_{R} B\left\langle\oplus_{R}(A \oplus \cdots \oplus A)\right.$.

Let $\mathfrak{M}_{T}\left({ }_{T} \mathfrak{M}\right)$ denote the category of right (left) $T$-modules and let $\mathfrak{M}_{S}\left(s_{\mathfrak{M}}\right)$ denote the category of right (left) $S$-modules. For $M \in \mathfrak{M}_{T}$ define $\boldsymbol{F}(M)=M \otimes_{T} \operatorname{Hom}\left({ }_{R} B,{ }_{R} A\right) \in \mathfrak{M}_{S}$ and for $N \in \mathfrak{M}_{S}$ define $\boldsymbol{G}(N)=N \otimes_{S}$ Hom $\left({ }_{R} A,{ }_{R} B\right) \in \mathfrak{M}_{T}$. Then $\boldsymbol{F}$ and $\boldsymbol{G}$ are covariant additive functor from $\mathfrak{M}_{T}$ to $\mathfrak{M}_{S}$ and from $\mathfrak{M}_{S}$ to $\mathfrak{M}_{T}$ respectively. Similarly we define $\boldsymbol{F}^{\prime}$ and $\boldsymbol{G}^{\prime}$ as follows. If $M^{\prime} \in{ }_{T} \mathfrak{M}$ define $\boldsymbol{F}^{\prime}\left(M^{\prime}\right)=\operatorname{Hom}\left({ }_{R} A,{ }_{R} B\right) \otimes_{T} M^{\prime} \in{ }_{S} \mathfrak{M}$ and if $N^{\prime} \in{ }_{S} \mathfrak{M}$ define $\boldsymbol{G}^{\prime}\left(N^{\prime}\right)=\operatorname{Hom}\left({ }_{R} B,{ }_{R} A\right) \otimes_{S} N^{\prime} \in{ }_{T} \mathfrak{M}$. From Theorem 1.2 and Proposition 1. 1 we obtain

Proposition 1.3. If ${ }_{R} B \leftrightarrow_{R}(A \oplus \cdots \oplus A)$ then $\boldsymbol{G}$ and $\boldsymbol{G}^{\prime}$ are exact functors while $\boldsymbol{F}$ and $\boldsymbol{F}^{\prime}$ preserve the projectivity. Furthermore $\boldsymbol{G} \cdot \boldsymbol{F} \cong 1 \mathfrak{m}_{T}$ and $\boldsymbol{G}^{\prime} \cdot \boldsymbol{F}^{\prime}=1_{T} \mathfrak{m}$.

Let $I(S)$ denote the set of two-sided ideals of $S$ and similarly for $I(T)$. If $\mathfrak{a} \in I(S)$ define $\left(\boldsymbol{G}^{\prime}, \boldsymbol{G}\right)(\mathfrak{a})=\operatorname{Hom}\left({ }_{R} B,{ }_{R} A\right) \cdot \mathfrak{a} \cdot \operatorname{Hom}\left({ }_{R} A,{ }_{R} B\right) \in I(T)$ and if $\mathfrak{b} \in I(T)$ define $\left(\boldsymbol{F}^{\prime}, \boldsymbol{F}\right)(\mathfrak{b})=\operatorname{Hom}\left({ }_{R} A,{ }_{R} B\right) \cdot \mathfrak{b} \cdot \operatorname{Hom}\left({ }_{R} B,{ }_{R} A\right) \in I(S)$. Then by Proposition 1. 1 we obtain

Proposition 1. 4. The mappings $\left(\boldsymbol{F}^{\prime}, \boldsymbol{F}\right)$ and $\left(\boldsymbol{G}^{\prime}, \boldsymbol{G}\right)$ preserve the inclusion. 
If ${ }_{R} B\left\langle\Theta_{R}(A \oplus \cdots \oplus A)\right.$, then $\left(\boldsymbol{F}^{\prime}, \boldsymbol{F}\right)$ preserves the product and $\left(\boldsymbol{G}^{\prime}, \boldsymbol{G}\right) \cdot\left(\boldsymbol{F}^{\prime}, \boldsymbol{F}\right)$ is the identity of $I(T)$.

From the above propositions, if we assume both ${ }_{R} B\left\langle\oplus_{R}(A \oplus \cdots \oplus A)\right.$ and ${ }_{R} A\left\langle\oplus{ }_{R}(B \oplus \cdots \oplus B)\right.$ then we have the following theorem.

Theorem 1. 5. If ${ }_{R} B\left\langle\oplus_{R}(A \oplus \cdots \oplus A)\right.$ and ${ }_{R} A\left\langle\oplus_{R}(B \oplus \cdots \oplus B)\right.$, then we have:

(i) $\operatorname{Hom}\left({ }_{R} A,{ }_{R} B\right)$ is a finitely generated projective generator as a left $S$ module and is the same as a right T-module. $\operatorname{Hom}\left({ }_{R} B,{ }_{R} A\right)$ is a finitely generated projective generator as a right $S$-module and is the same as a left T-module.

(ii) $\boldsymbol{G} \cdot \boldsymbol{F}$ and $\boldsymbol{F} \cdot \boldsymbol{G}$ are isomorphic to the identity functor of $\mathfrak{M}_{T}$ and $\mathfrak{M}_{S}$ respectively, i.e., $\mathfrak{M M}_{S}$ and $\mathfrak{M M}_{T}$ are equivalent. Similarly ${ }_{S} \mathfrak{M}$ and ${ }_{T} \mathfrak{M}$ are equivalent.

(iii) There is a one to one correspondence between $I(S)$ and $I(T)$ which preserves inclusion and one-sided projectivity.

(iv) For $M \in \mathfrak{M}_{T}$ the map $\xi \otimes f \longrightarrow \xi(f)$ from $\operatorname{Hom}\left(\operatorname{Hom}\left({ }_{R} A,{ }_{R} B\right)_{T}, M_{T}\right)$ $\otimes_{S} \operatorname{Hom}\left({ }_{R} A,{ }_{R} B\right)$ to $M$ is an isomorphism.

Similarly we have isomorphisms

$\operatorname{Hom}\left(\operatorname{Hom}\left({ }_{R} B,{ }_{R} A\right)_{S}, N_{S}\right) \otimes_{T} \operatorname{Hom}\left({ }_{R} B,{ }_{R} A\right) \cong N$ for $N \in \mathfrak{M}_{S}$, $\operatorname{Hom}\left({ }_{R} B,{ }_{R} A\right) \otimes_{S} \operatorname{Hom}\left({ }_{T} \operatorname{Hom}\left({ }_{R} B,{ }_{R} A\right),{ }_{T} M^{\prime}\right)^{\top} \cong M^{\prime}$ for $M^{\prime} \in{ }_{T} \mathfrak{M}$, $\operatorname{Hom}\left({ }_{R} A,{ }_{R} B\right) \otimes{ }_{T} \operatorname{Hom}\left({ }_{S} \operatorname{Hom}\left({ }_{R} A,{ }_{R} B\right),{ }_{S} N^{\prime}\right) \cong N^{\prime}$ for $N^{\prime} \in{ }_{S} \mathfrak{M}$.

The proof of (iv). By virtue of Proposition 1. 3 if $M \in \mathfrak{M}_{T}$ then $M \cong M \otimes_{T} \operatorname{Hom}\left({ }_{R} B,{ }_{R} A\right) \otimes_{S} \operatorname{Hom}\left({ }_{R} A,{ }_{R} B\right)$. Since ${ }_{R} A\left\langle\Theta_{R}(B \oplus \cdots \oplus B)\right.$, $\operatorname{Hom}\left({ }_{R} B,{ }_{R} A\right) \cong \operatorname{Hom}\left(\operatorname{Hom}\left({ }_{R} A,{ }_{R} B\right)_{T}, T_{T}\right)$ by Theorem 1.2 (i). Since $\operatorname{Hom}\left({ }_{R} A,{ }_{R} B\right)$ is finitely generated and projective as a right $T$-module, $M \otimes_{T} \operatorname{Hom}\left(\operatorname{Hom}\left({ }_{R} A,{ }_{R} B\right)_{T}, T_{T}\right) \cong \operatorname{Hom}\left(\operatorname{Hom}\left({ }_{R} A,{ }_{R} B\right)_{T}, M_{T}\right)$. Therefore we obtain an isomorphism $M \cong \operatorname{Hom}\left(\operatorname{Hom}\left({ }_{R} A,{ }_{R} B\right)_{T}, M_{T}\right) \otimes_{S} \operatorname{Hom}\left({ }_{R} A,{ }_{R} B\right)$ and this is given by $\xi \otimes f \longrightarrow \xi(f)$. Each of the statements can be proved by a similar way.

\section{\$2. A type of separable extension}

Let $\Lambda$ be a ring with the identity and $\Gamma$ a subring of $\Lambda$ having the same identity. Consider the two-sided $\Lambda$-modules $\Lambda \underset{\Gamma}{\otimes} \Lambda$ and $\Lambda$. Then we can easily check the following identifications: 
$\operatorname{Hom}_{(\Lambda, \Lambda)}(\Lambda, \Lambda)=C$ (two-sided $\Lambda$-homomorphisms) the center of $\Lambda$. $\operatorname{Hom}_{(\Lambda, \Lambda)}(\Lambda \underset{\Gamma}{\otimes} \Lambda, \Lambda \underset{\Gamma}{\otimes}, \Lambda)=(\Lambda \underset{\Gamma}{\otimes} \Lambda)^{\Gamma}=\{\xi \in \Lambda \underset{\Gamma}{\otimes} \Lambda \mid \gamma \xi=\xi \gamma$, for all $\gamma \in \Gamma\}$. $\operatorname{Hom}_{(\Lambda, \Lambda)}(\Lambda \underset{\Gamma}{\otimes} \Lambda, \Lambda)=\Lambda^{\Gamma}$, the centralizer of $\Gamma$ in $\Lambda$ and denote it by $\Delta$. $\operatorname{Hom}_{(\Lambda, \Lambda)}(\Lambda, \Lambda \underset{\Gamma}{\otimes} \Lambda)=(\Lambda \underset{\Gamma}{\otimes} \Lambda)^{\Lambda}$.

Now we assume that ${ }_{\Lambda} \Lambda \underset{\Gamma}{\otimes} \Lambda_{\Lambda}\left\langle\oplus{ }_{\Lambda}(\Lambda \oplus \cdots \oplus \Lambda)_{\Lambda}\right.$. Applying the conclusions of Theorem 1.2 in $\$ 1$, we have the following:

(1) $\Delta$ is finitely generated and projective as a $C$-module.

(2) $\Lambda \underset{\Gamma}{\otimes} \Lambda$ is isomorphic to $\operatorname{Hom}_{C}(\Delta, \Lambda)$ as a two-sided $\Lambda$-module.

Since $C$ is contained in $\Delta, \Delta$ is $C$-faithful. Therefore by Proposition A. 3 [1], (1) implies that $\Delta$ is a generator as a $C$-module. Then it is easily proved that $C$ is a direct summand of $\Delta$ as a $C$-module.

Next proposition is a generalization of Proposition 2. 2 [2].

Proposition 2. 1. Let $A$ be a ring with the center $C$ and let $\Delta$ be a subring of $\Lambda$ containing $C$. Define a two-sided $\Lambda$-epimorphism $\varphi_{0}: \operatorname{Hom}_{C}(\Delta, \Lambda) \longrightarrow \Lambda$ by $\varphi_{0}(f)=f(1) . \quad$ Then there exists a two-sided $\Lambda$-homomorphism $\psi_{0}: \Lambda \longrightarrow \operatorname{Hom}_{C}(\Lambda, \Lambda)$ such that $\varphi_{0} \cdot \psi_{0}=1_{A}$ if and only if $C$ is a $C$-direct summand of $\Delta$.

Proof. Assume the existence of $\psi_{0}$. If we take $g=\psi_{0}(1)$ then $\lambda \cdot g=g \cdot \lambda$ for all $\lambda \in A$. Therefore $\lambda g(\delta)=g(\delta) \lambda$ for all $\delta \in \Delta$ and so $g(\delta) \in C$. This implies that $g \in \operatorname{Hom}_{C}(\Delta, C)$. Since $g(1)=1, g(c)=c g(1)=c$ for $c \in C$, and $C$ is a direct summand of $\Delta$. Conversely if $C$ is a direct summand of $\Delta$ and if $p$ is the projection from $\Delta$ to $C$, then $p(\delta) \in C$ for all $\delta \in \Delta$ and $p(1)=1$. Therefore $\lambda p(\delta)=p(\delta) \lambda$ for all $\lambda \in \Lambda$. If we define the homomorphism $\psi_{0}: \Lambda \longrightarrow \operatorname{Hom}_{C}(\Delta, \Lambda)$ by $\varphi_{0}(\lambda)=\lambda \cdot p=p \cdot \lambda$, then $\varphi_{0} \cdot \psi_{0}(\lambda)=$ $(\lambda \cdot p)(1)=\lambda \cdot p(1)=\lambda$. This completes the proof.

With these preparations we have the following theorem.

Theorem 2. 2. Let $A$ be a ring with the center $C, \Gamma$ a subring of $\Lambda$, and let $\Delta$ be the centralizer of $\Gamma$ in $\Lambda$. If $\Lambda \underset{\Gamma}{\otimes}$ is isomorphic to a two-sided $\Lambda$ direct summand of a finite direct sum of copies of $\Lambda$, then $\Delta$ is $C$-finitely generated projective and $\Lambda$ is a separable extension of $\Gamma$.

Proof. We have the following commutative diagram 


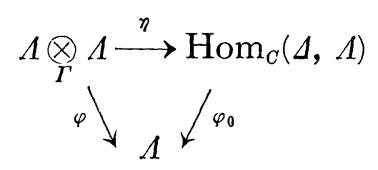

where $\eta(x \otimes y)(\delta)=x \delta y, \quad \varphi(x \otimes y)=x y$ and $\varphi_{0}$ is the homomorphism given in Proposition 2. 1. Since, as we have remarked above, $C$ is a $C$-direct summand of $\Delta$, there exists $\psi_{0}: \Lambda \longrightarrow \operatorname{Hom}_{C}(\Delta, \Lambda)$ such that $\varphi_{0} \cdot \psi_{0}=1_{\Lambda}$. Since $\eta$ is a two-sided $\Lambda$-isomorphism if we define $\phi: \Lambda \rightarrow \Lambda \underset{\Gamma}{\otimes} \Lambda$ by $\psi=\eta^{-1} \cdot \psi_{0}, \quad \psi$ is a two-sided $\Lambda$-homomorphism and $\varphi \cdot \psi=1_{\Lambda}$. Therefore $\Lambda$ is a separable extension of $\Gamma$.

The separable extension treated in Theorem 2.2 is a generalization of the notion of central separable algebras. If $\Gamma$ is the center of $\Lambda$ and $\Lambda$ is a separable $\Gamma$-algebra then $\Lambda$ is a projective generator as a left $\Lambda \underset{\Gamma}{\otimes} \Lambda^{0}$ module and so $\Lambda \underset{\Gamma}{\otimes} \Lambda$ is isomorphic to a direct summand of a finite direct sum of copies of $A$. In the following section we discuss a type of separable extension satisfying this condition.

Remark. The condition $A \underset{\Gamma}{\otimes} \Lambda\langle\oplus \oplus \oplus \cdots \oplus \Lambda$ is equivalent to that there exist $f_{i} \in \operatorname{Hom}_{(\Lambda, \Lambda)}(\Lambda, \Lambda \underset{\Gamma}{\otimes} \Lambda)$ and $x_{i} \in \Delta$ such that $\sum f_{i}\left(x_{i}\right)=1 \otimes 1 \in \Lambda \underset{\Gamma}{\otimes} \Lambda$.

\section{§3. A further type of separable extension}

Proposition 3. 1. Let $\Delta$ be an R-central separable algebra and let $\Gamma$ be an $R$-algebra with the center $C . \quad$ Then the center of $A=\Gamma \underset{R}{\otimes} \Delta$ is $C$.

Proof. Since $\Delta$ is $R$-projective, the exact sequence

$$
0 \longrightarrow \mathrm{C} \longrightarrow \Gamma
$$

yields the exact sequence

$$
0 \longrightarrow C \underset{R}{\otimes} \underset{R}{\longrightarrow} \Delta \underset{R}{\underset{\otimes}{\otimes}} \Delta .
$$

If we put $\Delta_{1}=C \underset{R}{\otimes} \Delta$ then $\Delta_{1}$ is a $C$-central separable algebra contained in A. Since $R$ is a direct summand of $\Delta, \Gamma$ may be considered as a subring of $\Lambda$. Let $\Lambda^{\Delta_{1}}$ be the centralizer of $\Delta_{1}$ in $\Lambda$. Then $\Lambda^{\Lambda_{1}}$ contains $\Gamma$ and $\Lambda^{\Delta_{1}} \underset{C}{\otimes} \Delta_{1} \cong \Lambda$ by the map $x \otimes y \longrightarrow x y$ ([2], Theorem 3.3). The exact sequence

$$
0 \longrightarrow \Gamma \longrightarrow \Lambda^{\Delta_{1}} \longrightarrow \Lambda^{\Delta_{1}} / \Gamma \longrightarrow 0
$$


yields the exact sequence

$$
0 \longrightarrow \Gamma \otimes_{C} \Delta_{1} \longrightarrow \Lambda^{\Delta_{1}} \otimes_{C} \Delta_{1} \longrightarrow\left(\Lambda^{\Delta_{1}} / \Gamma\right) \otimes_{C} \Delta_{1} \longrightarrow 0
$$

The first two terms of the above sequence are equal to $\Lambda$. Therefore $\left(\Lambda^{\Delta_{1}} / \Gamma\right) \underset{C}{\otimes} \Delta_{1}=0$ and $\Lambda^{\Delta_{1}}=\Gamma$. Thus we have

$$
\text { center } \Lambda \subseteq \text { center } \Lambda^{\Lambda_{1}}=\text { center } \Gamma=C \subseteq \text { center } \Lambda \text {. }
$$

This completes the proof.

We remark that

$$
C=\text { center } \Delta_{1}=\Delta_{1} \cap \Lambda^{\Delta_{1}}=\Delta_{1} \cap \Gamma .
$$

In Proposition 3.1 $A=\Gamma \otimes_{R} \Delta$ is a separable extension of $\Gamma$ by Cor. 2. 8, [5]. Proposition 3. 1 shows that when we consider the separable extension of this type we may suppose that $A=\Gamma \underset{C}{\otimes_{C}} \Delta$ where $C$ is the common center of $\Gamma, \Delta$ and $A$, and, $\Delta$ is a central $C$-separable algebra. In this section we discuss separable extensions of this type.

Proposition 3. 2. Let $\Lambda=\Gamma \underset{\mathrm{C}}{\otimes} \Delta$ be a separable extension of $\Gamma$ treated above. Then we have:

(i) The centralizer of $\Gamma$ in $\Lambda$ is equal to $\Delta$.

(ii) $\Lambda$ is left (resp. right) $\Gamma$-finitely generated projective and $\Gamma$ is a left (resp. right) $\Gamma$-direct summand of $\Lambda$.

Proof. By the proof of Proposition 3. 1 we know that $\Lambda^{4}=\Gamma$. If we put $\Lambda^{\Gamma}=\Delta^{\prime}$ then $\Delta^{\prime} \supseteq \Delta$. Since $C$ is the center of $\Gamma$ we have $C=\Gamma \cap \Delta^{\prime}$. On the other hand $\Delta^{\prime} \Delta=\Delta^{\prime} \cap \Delta^{\Delta}=\Delta^{\prime} \cap \Gamma=C$. Therefore by [2] Theorem 3. 3 we have $\Delta^{\prime}=\Delta^{\prime} \Delta \otimes_{C} \Delta=C \otimes_{C} \Delta=\Delta$. This completes the proof of (i).

Since $\Delta$ is a central $C$-separable algebra, $\Delta$ is $C$-finitely generated projective and $C$ is a direct summand of $\Delta$. Therefore (ii) is clear.

Let $\Lambda^{\mathfrak{M}} \mathbb{M}_{\Lambda}\left(\mathfrak{M}_{\Gamma}\right)$ denote the category of two-sided $\Lambda$ - $(\Gamma$-) modules. For $M \in{ }_{A} \mathfrak{M}_{A}$ define $\boldsymbol{S}(M)=\operatorname{Hom}_{(\Delta, \Delta)}(\Delta, M)=M^{\Delta}$. Then it is easily checked that $M^{\Delta} \in \Gamma_{\Gamma} \mathfrak{M}_{\Gamma} . \quad$ For $\quad N \in \Gamma_{\Gamma} \mathfrak{M}_{\Gamma}$ define $\quad \boldsymbol{T}(N)=N \underset{C}{\otimes} \Delta . \quad N \underset{C}{\otimes} \Delta$ is a twosided $A$-module. Since $\Delta$ is central $C$-separable $\boldsymbol{T} \cdot \boldsymbol{S}(M)=M^{\Delta} \underset{C}{\otimes_{C} \Delta}$ is isomorphic to $M$ as a two-sided $\Delta$-module ([2], Theorem 3.1). As is easily seen it is a two-sided $\Lambda$-isomorphism. On the other hand since $\Delta$ is $\Delta \underset{C}{\otimes} \Delta^{0}$ projective we have isomorphisms $\boldsymbol{S} \cdot \boldsymbol{T}(N)=\operatorname{Hom}_{(\Delta, \Delta)}\left(\Delta, N \underset{C}{\otimes_{C}} \Delta\right) \cong N \underset{C}{\otimes_{C}}$ 
$\operatorname{Hom}_{(\Delta, \Delta)}(\Delta, \Delta) \cong N \underset{C}{\otimes} C \cong N$ as $C$-modules. Furthermore these are twosided $\Gamma$-isomorphisms. Thus we have

Proposition 3. 3. Under the same assumption as in Proposition 3. $2{ }_{A}^{\mathfrak{M R}_{A}}$ is equivalent to ${ }_{\Gamma} \mathrm{M}_{\Gamma}$.

Since $\Delta$ is central $C$-separable, $\Delta$ is a generator as a left $\Delta \otimes_{C} \Delta^{0}$-module. So $\Delta \otimes_{C} \Delta$ is a direct summand of a finite direct sum of copies of $\Delta$ as a two-sided $\Delta$-module. Therefore as is easily seen $\Gamma \otimes_{C} \Delta \otimes_{C} \Delta=\left(\Gamma \otimes_{C} \Delta\right) \otimes_{\Gamma}\left(\Gamma \otimes_{C} \Delta\right)$ is a direct summand of a finite direct sum of copies of $\Gamma \otimes_{C} \Delta$ as a twosided $\Gamma \underset{C}{\otimes} \Delta$-module. Therefore we have

Proposition 3. 4. Let $\Delta$ be a central C-separable algebra. Then, for the separable extension $\Lambda=\Gamma \underset{C}{\otimes} \Delta$ of $\Gamma$ treated above, the two-sided 1 -module $\Lambda \underset{\Gamma}{\otimes} \Lambda$ is a direct summand of a finite direct sum of copies of $\Lambda$.

\section{REFERENCES}

[ 1 ] M. Auslander and O. Goldman, Maximal orders, Trans. Amer. Math. Soc., 97 (1960), $1-24$.

[2 ] M. Auslander and O. Goldman, The Brauer group of a commutative ring, Trans. Amer. Math. Soc., 97 (1960), 367-409.

[ 3 ] H. Bass, The Morita theorems, Lecture notes, Summer Inst. on Algebra 1962, Univ. of Oregon.

[ 4] H. Cartan and S. Eilenberg, Homological algebra, Princeton, 1956.

[5] K. Hirata and K. Sugano, On semisimple extensions and separable extensions over non commutative rings, J. Math. Soc. Japan, 18 (1966), 360-373.

[6] K. Morita, Duality for modules and its applications to the theory of rings with minimum conditions, Sci. Rep. Tokyo Kyoiku Daigaku, Sect. A, 6 No. 150 (1958), 83-142.

[7] G. Azumaya, Completely faithful modules and self-injective rings, Nagoya Math. J., 27 (1966), 697-708.

Yamanashi University 